\title{
Yersinia enterocolitica enteritis versus other bacterial enteritis in ultrasonic findings: a single- center case control study
}

\section{Eri Miyata}

Juntendo University Faculty of Medicine

Keisuke Jimbo ( $\nabla$ kjinbo@juntendo.ac.jp )

Juntendo University Faculty of Medicine https://orcid.org/0000-0001-6317-4305

Reiko Kyoudo

Juntendo University Faculty of Medicine

Takahiro Kudo

Juntendo University Faculty of Medicine

Toshiaki Shimizu

Juntendo University Faculty of Medicine

Research article

Keywords: abdominal ultrasonography, C-reactive protein, mesenteric lymphnode, pericecal hyperechoic region, terminal ileum

Posted Date: March 4th, 2020

DOI: https://doi.org/10.21203/rs.3.rs-16048/v1

License: (c) (i) This work is licensed under a Creative Commons Attribution 4.0 International License.

Read Full License 


\section{Abstract}

Background: Yersinia enterocolitica $(Y e)$ causes enteritis with mesenteric lymphadenitis and terminal ileitis, and can be difficult to distinguish from acute appendicitis, especially in children. In addition, because detection from stool culture is more difficult for Ye than for other bacteria causing enteritis, the diagnosis of Ye enteritis is not easy. Abdominal ultrasonography is useful for diagnosing bacterial enteritis, including Ye enteritis. This study diagnosed Ye enteritis and other bacterial enteritis by stool culture and compared and analyzed the ultrasonic findings and clinical features in children. The aims of this study are to compare and analyze ultrasonic findings and clinical features in children with $Y e$ enteritis and other bacterial enteritis.

Methods: Participants in this retrospective study comprised all 34 bacterial enteritis patients (3-18 years old) treated between 2014 and 2017. Patients were divided into the Yersinia enterocolitica enteritis (Ye) group and other bacterial enteritis (non-Ye) group from whom other pathogens were detected. Ultrasonic findings (including maximum and minimum diameters of ileocecal lymph nodes (C-LNs), mean major/minor axis ratio, wall thickness of the terminal ileum, and presence of a pericecal hyperechoic region) and blood examinations at first visit were compared between two groups.

Results: The Ye group included 13 patients and the non-Ye group included 21 patients. Mean C-reactive protein level was higher in the Ye group $(5.9 \pm 3.0 \mathrm{mg} / \mathrm{dL})$ than in the non-Ye group $(2.7 \pm 3.4 \mathrm{mg} / \mathrm{dL}, p=$ 0.049). No difference in mean maximum diameter of C-LNs was seen between two groups. However, mean C-LN major/minor axis ratio was lower in the Ye group than in the non-Ye group $(p=0.002)$ and terminal ileal wall thickness value was higher in the Ye group than in the non-Ye group $(p=0.004)$. Pericecal hyperechoic region was more frequent in the Ye group than in the non-Ye group $(p=0.021)$.

Conclusion: The combination of characteristic ultrasonic findings identified in this study may improve ultrasonic differentiation of Yersinia enterocolitica enteritis and other bacterial enteritis.

\section{Background}

Yersinia species, among which Y. pestis, Y. enterocolitica (Ye), and Y. pseudotuberculosis mainly infect humans, are Gram-negative anaerobic bacilli that show optimal growth in cold culture at $0-4{ }^{\circ} \mathrm{C}$ [1]. Ye causes enteritis with mesenteric lymphadenitis and terminal ileitis. Ye enteritis has an estimated prevalence of about $1 \%$ of bacterial enteritis in developed countries [2] and is frequently difficult to distinguish from acute appendicitis [3, 4]. In addition, because detection from stool culture is more difficult for Ye than for other bacteria causing enteritis, the diagnosis of Ye enteritis is not easy at present, although the accuracy of Ye diagnosis is slightly increased by combining serological tests with stool cultures $[5,6]$. On the other hand, abdominal ultrasonography is performed for the diagnosis of bacterial enteritis, including Ye enteritis, and mesenteric lymph node swelling and terminal ileitis have been reported in patients regardless of bacterial species [7]. However, no studies comparing differences in ultrasonic findings between Ye enteritis and other bacterial enteritis have been reported. This study 
compared and analyzed the ultrasonic findings and clinical features between Ye enteritis and other bacterial enteritis diagnosed from stool culture.

\section{Methods}

\section{Study design}

This was a retrospective case control study to assess the ultrasonic findings and clinical features in children with bacterial enteritis, including Ye infection.

\section{Patients}

This study was carried out as part of the "Technology development and examination of intestinal blood flow quantification in child abdominal ultrasonography" study at Juntendo University (approved by the institutional ethics committee, Faculty of Medicine, Juntendo University, Japan as No. 13-072). In this retrospective investigation, data for all patients aged between 3 to 18 years old at the time of treatment and who were eligible according to the inclusion criteria were obtained from the electronic medical records of pediatric outpatient and emergency room of the Department of Pediatrics, Faculty of Medicine, Juntendo University, Japan between 2014 and 2017. Detailed medical histories were obtained from the parents of all patients when ultrasonography was performed at the first visit. The inclusion criteria of this study were 1) suffering from fever and gastrointestinal symptoms such as abdominal pain, diarrhea, and vomiting; 2) detection of any pathogen from stool culture; and 3) performance of abdominal ultrasonography at the first visit. The exclusion criteria of this study were 1) previous abdominal surgery; 2) history of bacterial enteritis or other digestive diseases diagnosed by pediatricians; 3 ) chronic medical history of recurrent abdominal pain or diarrhea; 4) growth retardation on the growth curve; 5) administration of antibiotics or any other drugs at the first visit; or 6) cases with no clear ultrasonic images available for analysis.

\section{Subject preparation}

Patients were divided into two groups according to the results of stool cultures: a Ye group, in which Ye enteritis was detected; and a non-Ye group, in which enteritis was attributed to other pathogens such as Campylobacter jejuni, Salmonella, and Escherichia coli. Clinical features, inflammatory markers like WBC and C-reactive protein (CRP), and abdominal ultrasonic findings were compared between groups. Assessment of abdominal ultrasonography

Abdominal ultrasonography was performed in all children. All ultrasonic examinations were performed by two pediatricians (Jimbo K and Miyata E), with 14 and 8 years of experience in pediatric ultrasonic, respectively, using a HI VISION Preirus ${ }^{\circledR}$ system (Hitachi-Aloka Medical, Tokyo, Japan) and an 8- to $12-\mathrm{MHz}$ high-frequency linear transducer (EUP-L54MA ${ }^{\circledR}$; Hitachi-Aloka Medical). The ultrasonic findings were accepted when the interpretations of the two sonographers matched. Ultrasonic studies were performed when the patients were either not moving or sleeping, to minimize ultrasonic artifacts. 
Abdominal ultrasonic findings were assessed using the following three parameters (Fig. 1) and the results were recorded in a data sheet:

1) Measurement of maximum and minimum diameter in five randomly selected ileocecal lymph nodes (C-LNs) and calculation of mean major/minor axis ratio of the five C-LNs.

2) Measurement of wall thickness of the terminal ileum. The terminal ileum was defined as the ileum exiting within $5 \mathrm{~cm}$ from ileocecal valve on the oral side, and wall thickness of the ileum was defined as abnormal thickening for values $>3.0 \mathrm{~mm}$. Thickness was measured by placing a pointer between the mucosal surface and the serosa in the bowel loop [8].

3) Detection of the presence of a pericecal hyperechoic region. A pericecal hyperechoic lesion was defined as a cecal and terminal ileal extraluminal hyperechoic band thicker than $4.0 \mathrm{~mm}$, which was defined from the bowel serosa to the edge of the hyperechoic band, and clearly distinguishable from the terminal ileum and thickened intestinal tract around the ileocecal region.

\section{Statistical analysis}

BellCurve for Excel software (Social Survey Research Information, Tokyo, Japan) was used to perform statistical analysis. Continuous variables are presented as mean value, standard deviation (SD) and range or median value, as appropriate. Categorical variables are expressed as counts (percentage). The chi-squared test was used for comparisons with more than five categorical variables. The nonparametric Mann-Whitney U test was used to compare data showing skewed distributions. Values of $p<0.05$ were considered statistically significant for blood tests and abdominal ultrasonic findings between the Ye and non-Ye groups. Furthermore, receiver operating characteristic (ROC) analysis was performed for maximum diameter, mean long-short diameter ratio of C-LNs, and wall thickness of the terminal ileum, and significant differences between the respective areas under the curve (AUCs) were analyzed. The point closest to the point $(0,1)$ on the ROC curve was taken as the cut-off value, and sensitivity and specificity were calculated. The statistical methods of this study were reviewed by Nobuaki Matsunaga, MD, PhD, from the Antimicrobial Resistance Clinical Reference Center, National Center for Global Health and Medicine Hospital.

\section{Results}

A total of 34 patients were included in this study. Mean age for all patients was $9.1 \pm 2.9$ years. The 34 cases comprised $13(38.2 \%)$ cases of Ye enteritis, $10(29.4 \%)$ cases of C. jejuni enteritis, $9(26.4 \%)$ cases of Salmonella enteritis, and $2(5.8 \%)$ cases of E. coli (0157) enteritis as diagnosed by stool culture (Additional file 1: Table S1). Mean age showed no significant difference between the Ye group (10.2 \pm 2.6 years $)$ and non-Ye group $(9.0 \pm 3.1$ years, $p=0.31)$. Sex ratio likewise did not differ significantly between the Ye group ( 7 boys, 6 girls) and the non-Ye group ( 9 boys, 12 girls; $p=0.53$ ). In terms of gastrointestinal symptoms, the Ye and non-Ye groups showed abdominal pain in 12 of 13 cases (92.3\%) and 20 of 21 cases (95.2\%), diarrhea in 6 of 13 cases (46.1\%) and 11 of 21 cases (52.3\%), and vomiting 
in 3 of 13 cases (23.1\%) and 4 of 21 cases (19.0\%) cases, respectively, with no significant differences in clinical symptoms between groups $(p=0.72, p=0.72, p=0.77$, respectively).

In laboratory test, mean WBCs in the Ye and non-Ye groups were 12,310 $\pm 4,703 / \mu \mathrm{L}$ and 10,200 $\pm 3,454$ / $\mu \mathrm{L}$, with no significant difference between groups $(p=0.12)$. Mean CRP values of Ye and non-Ye groups were $5.9 \pm 3.0 \mathrm{mg} / \mathrm{dL}$ and $2.7 \pm 3.4 \mathrm{mg} / \mathrm{dL}$, showing a significant difference between groups $(p=0.049)$ (Table 1).

A summary of the actual measured values from ultrasonography is shown in Table 1. C-LN maximum diameter was $11.2(7.5-17.7) \mathrm{mm}$ in the Ye group and $10.3(3.0-21.6) \mathrm{mm}$ in the non-Ye group, with no significant difference between groups $(p=0.644)$. Mean C-LN major/minor axis ratio was significantly lower in the Ye group (1.40 (1.25-1.65)) than in the non-Ye group (1.62 (1.36-2.05); $p=0.002)$. Terminal ileal wall thickness was significantly higher in the Ye group $(6.5(3.5-8.9) \mathrm{mm})$ than in the non-Ye group $(5.0(2.5-8.1) \mathrm{mm} ; \mathrm{p}=0.004)$ (Fig. 2). The diagnostic performance of ultrasonic findings in differentiating Ye enteritis from non-Ye enteritis is summarized in Additional file 2: Table S2. Among C-LN maximum diameter, mean C-LN major-minor axis ratio, and wall thickness of the terminal ileum, mean C-LN majorminor axis ratio and wall thickness of the terminal ileum showed significantly higher AUCs for differentiating Ye enteritis from non-Ye enteritis than C-LN maximum diameter (AUC $=0.791 ; p<0.01$ and $0.818 ; p<0.01$, respectively) (Table 2). Furthermore, when cut-off values were set as the point closest to $(0,1)$ on the ROC curve (Fig. 3), sensitivities of C-LN maximum diameter, mean C-LN major-minor axis ratio, and wall thickness of the terminal ileum were $62.9 \%, 76.9 \%, 76.9 \%$, respectively, and specificities were $57.1 \%, 76.1 \%, 80.9 \%$, respectively (Table 2 ). In addition, the pericecal hyperechoic region was observed in 11 of 13 cases (84.6\%) in the Ye group and 8 of 21 cases $(38.0 \%)$ in the non-Ye group (Additional file 2: Table S2), representing a significant difference $(p=0.021)$. Sensitivity and specificity of this finding were $84.6 \%$ and $61.9 \%$, respectively. Furthermore, all cases with a pericecal hyperechoic region showed spontaneous resolution of this finding within several weeks after starting treatment for bacterial enteritis.

\section{Discussion}

In this study, no significant difference was could be found between the Ye and non-Ye groups in background or clinical symptoms of enrolled patients. However, both CRP and terminal ileal thickness were significantly higher in the Ye group than in the non-Ye group. A past study reported that Crohn's disease patients with terminal ileal lesions showed significantly higher CRP than ulcerative colitis patients with lesions localized only to the colon. Presence of terminal ileal lesions also correlated with high CRP value in comparisons between Crohn's disease patients [9]. Given that finding, the incidence of Ye group patients presenting with significantly higher CRP values in this study may have indirectly suggested that Ye enteritis developed predominantly in the terminal ileum.

Although this study mainly focused on differences in abdominal ultrasonic findings between Ye and nonYe enteritis, a previous abdominal ultrasonic study of Yersinia, Campylobacter, and Salmonella bacterial 
enteritis (total, 117 cases) reported that C-LNs of Yersinia cases were more swollen than those caused by other bacteria [10]. Moreover, another abdominal ultrasonic study of eight cases of Yersinia enteritis reported that wall thickness of the terminal ileum was $7-10 \mathrm{~mm}$ in all 8 cases $(100 \%)$ cases and swollen C-LNs $(7-21 \mathrm{~mm})$ were observed in 6 cases $(75.0 \%)$ [11]. On the other hand, this study found no difference in maximum diameter of $C$-LNs between the two groups $(p=0.65)$, despite the thicker wall of the terminal ileum in the Ye group compared to the non-Ye group $(p=0.004)$. However, this finding suggests that similar results were obtained for terminal ileal wall thickness with Ye enteritis, even though several decades have passed since the previous two reports and the performance of ultrasonic equipment has greatly improved.

In addition, this study compared the mean C-LN major-minor axis ratio of five C-LNs between Ye and nonYe groups, to indirectly evaluate the C-LN shape. Mean C-LN major-minor axis ratio was lower in the Ye group than in the non-Ye group $(p=0.002)$. Although we found no reports discussing ultrasonic C-LN shape in bacterial enteritis including Ye infection, mean C-LN major-minor axis ratio in the Ye group was closer to 1.0 than that in the non-Ye group in this study. The results of our ultrasonic examination of C-LN shape suggested that C-LNs in Ye enteritis had a rounder shape rather than an ellipsoid shape.

Further, we analyzed maximum diameter and mean long-short diameter ratio of C-LNs, and wall thickness of the terminal ileum by ROC analysis. The results for mean long-short diameter ratio and wall thickness of the terminal ileum showed almost the same AUCs and the sensitivity and specificity at the determined cut-offs were also largely comparable.

In addition, the frequency of pericecal hyperechoic regions was higher in the Ye group than in the non-Ye group $(p=0.021)$. This pericecal hyperechoic region resembled the "creeping fat" $[8,12]$ frequently observed in Crohn's disease patients and CRP values and wall thickness of the terminal ileum were higher in the Ye group than in the non-Ye group in this study. We therefore speculated that the echo density of ileal stroma might be enhanced such as creeping fat, because Ye enteritis involves very severe inflammation spreading from the entire ileal wall layer to the surrounding fatty tissue as in Crohn's disease. However, creeping fat is a frequent finding in Crohn's disease, but can also be found in another gastrointestinal diseases [13].

We thus suggest that abdominal ultrasonic evaluation of the long-short diameter ratio of C-LNs, wall thickness of the terminal ileum, and the pericecal hyperechoic region could improve the diagnostic rate for Ye enteritis.

This study showed some limitations. First, we included only small numbers of patients with Ye and nonYe enteritis. Although our institute treats many cases of bacterial enteritis in the outpatient department, cases in this study may have been reduced because relatively few patients might have had total findings from ultrasonic evaluations available at the time of the first outpatient visit. On the other hand, we should not consider information bias related to ultrasonic inspection, because we did not determine evaluation findings before study planning. Second, creation of clinical scores using the findings for long-short diameter ratio of C-LNs, wall thickness of the terminal ileum, and pericecal hyperechoic regions also 
appears important, but could not be addressed because of the small number of participating cases in this study.

\section{Conclusions}

Our study demonstrated, in Ye enteritis, C-LNs presented a rounder shape, the terminal ileal wall was thicker, and the frequency of a pericecal hyperechoic region was significantly higher than in other bacterial enteritis. Although the combination of these findings appears likely to improve the abdominal ultrasonic diagnosis rate for Ye enteritis, more cases of bacterial enteritis should be examined in the future to validate our findings.

\section{Abbreviations}

AUC

areas under the curve; C-LN:ileocecal lymph node; CRP:C-reactive protein; F:female; FPF:false positive fraction; M:male; P:positive; ROC:receiver operating characteristic; SD:standard deviation; TPF:true positive fraction; Ye:Y. enterocolitica

\section{Declarations}

\section{Ethics approval and consent to participate}

The study protocol was approved by approved by the institutional ethics committee, Faculty of Medicine, Juntendo University, Japan. Because this study was a retrospective study and all subjects were anonymized, informed consent was not required.

\section{Consent for publication}

The parents of the patients presented in the tables and figures used within this manuscript gave consent for their personal or clinical details along with any identifying images to be published in this study. Written and signed consent for publication was obtained from the parents of the patients.

\section{Availability of data and materials}

The datasets used and analysed during the current study are available from the corresponding author upon reasonable request.

\section{Competing interests}

The authors declare that they have no competing interests.

\section{Acknowledgements}

The authors would like to express their appreciation to the patients and their parents. 


\section{Funding}

The authors had no funding sources for this study.

\section{Authors' contributions}

$\mathrm{ME}, \mathrm{JK}$, and $\mathrm{KR}$ designed research. $\mathrm{ME}, \mathrm{JK}, \mathrm{KR}$, and $\mathrm{KT}$ treated patients and collected material and clinical data from patients. ME and JK performed the ultrasonographic study. JK, KT, and ST analyzed and interpreted data. ME drafted the manuscript. ST approved the final version of paper to be published. All authors read and approved the final manuscript.

\section{References}

1. Long SS, Pickering LK, Prober CG. Principles and practice of pediatric infectious diseases. In., 4th edn. Edinburgh ; New York: Elsevier Churchill Livingstone; 2012: 1 online resource (xxix, 1712 pages).

2. Iwamoto M, Huang JY, Cronquist AB, Medus C, Hurd S, Zansky S, Dunn J, Woron AM, Oosmanally N, Griffin PM, et al. Bacterial enteric infections detected by culture-independent diagnostic testsFoodNet, United States, 2012-2014. MMWR Morb Mortal Wkly Rep 2015;64(9):252-257.

3. Watanabe K, Watanabe N, Jin M, Matsuhashi T, Koizumi S, Onochi K, Sawaguchi M, Tawaraya S, Miyazawa $\mathrm{H}$, Uchinami $\mathrm{H}$, et al: Mesenteric lymph node abscess due to Yersinia enterocolitica: case report and review of the literature. Clin J Gastroenterol 2014;7(1):41-47.

4. Laji N, Bowyer R, Jeyaratnam D, Zuckerman M. Another mistaken case of appendicitis. BMJ Case Rep 2015;2015.

5. Savin C, Leclercq A, Carniel E. Evaluation of a single procedure allowing the isolation of enteropathogenic Yersinia along with other bacterial enteropathogens from human stools. PLoS One 2012;7(7):e41176.

6. Marks MI, Pai CH, Lafleur L, Lackman L, Hammerberg O. Yersinia enterocolitica gastroenteritis: a prospective study of clinical, bacteriologic, and epidemiologic features. J Pediatr 1980;96(1):26-31.

7. Puylaert JB. Ultrasound of acute GI tract conditions. Eur Radiol 2001; 11(10):1867-1877.

8. Siegel MJ. Pediatric sonography. In., 4th ed. Philadelphia: Lippincott Williams \& Wilkins; 2011.

9. Muller KE, Lakatos PL, Kovacs JB, Arato A, Varkonyi A, Nemes E, et al. Baseline Characteristics and Disease Phenotype in Inflammatory Bowel Disease. J Pediatr Gastroenterol Nutr 2016;62(1):50-55.

10. Puylaert JB, Van der Zant FM, Mutsaers JA. Infectious ileocecitis caused by Yersinia, Campylobacter, and Salmonella: clinical, radiological and US findings. Eur Radiol 1997;7(1):3-9.

11. Matsumoto T, lida M, Sakai T, Kimura Y, Fujishima M. Yersinia terminal ileitis: sonographic findings in eight patients. AJR Am J Roentgenol 1991;156(5):965-967.

12. Mao R, Kurada S, Gordon IO, Baker ME, Gandhi N, McDonald C, et al. The Mesenteric Fat and Intestinal Muscle Interface: Creeping Fat Influencing Stricture Formation in Crohn's Disease. Inflamm 
Bowel Dis 2019;25(3):421-426.

13. Golder WA. The "creeping fat sign"-really diagnostic for Crohn's disease? Int J Colorectal Dis 2009;24(1):1-4.

\section{Tables}

Due to technical limitations, tables are only available as a download in the supplemental files section

Figures
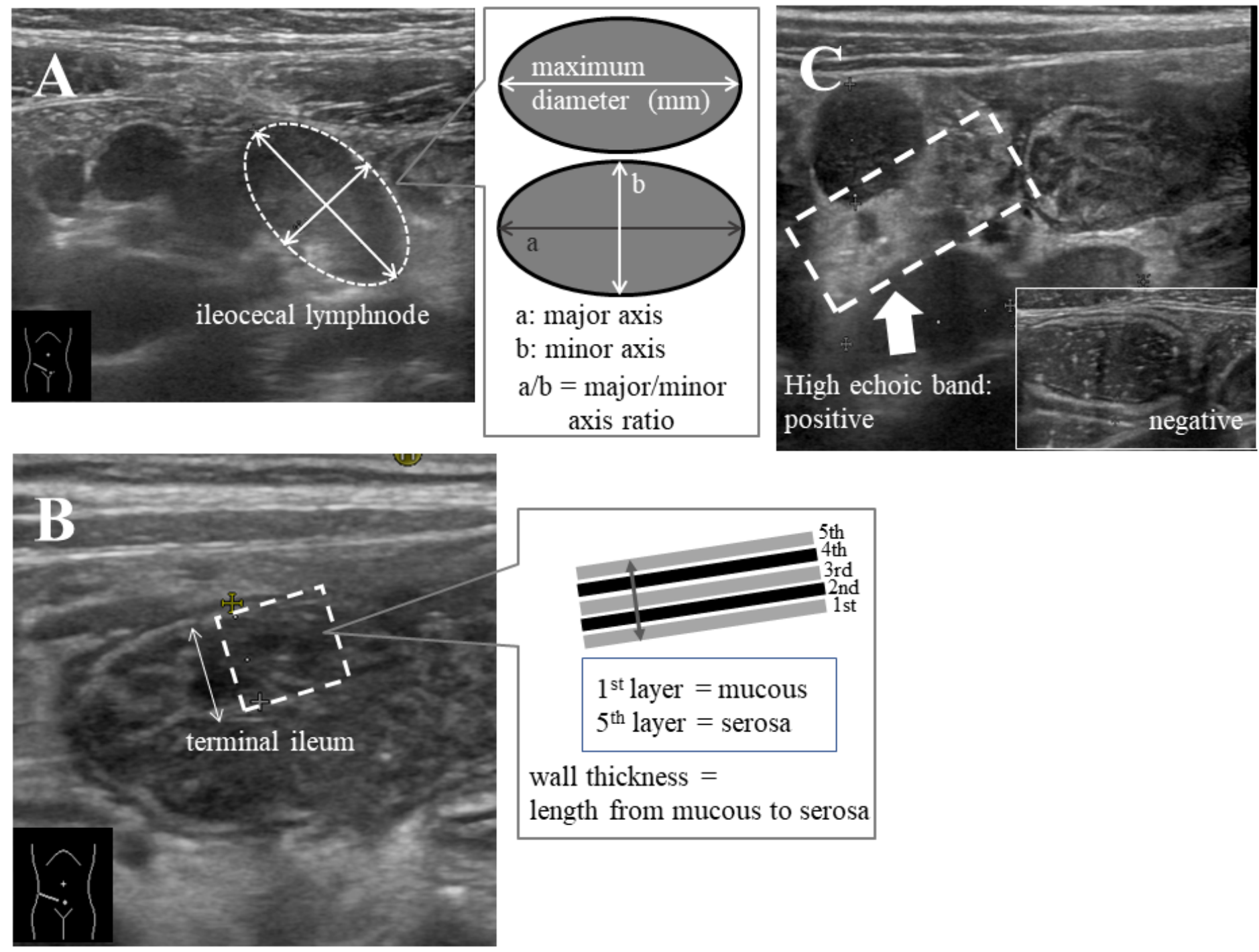

Figure 1

Three parameters of abdominal ultrasonic study. a Maximum diameter and major/minor axis ratio of ileocecal lymph nodes; $b$ Wall thickness of the terminal ileum; c Pericecal high echoic band 


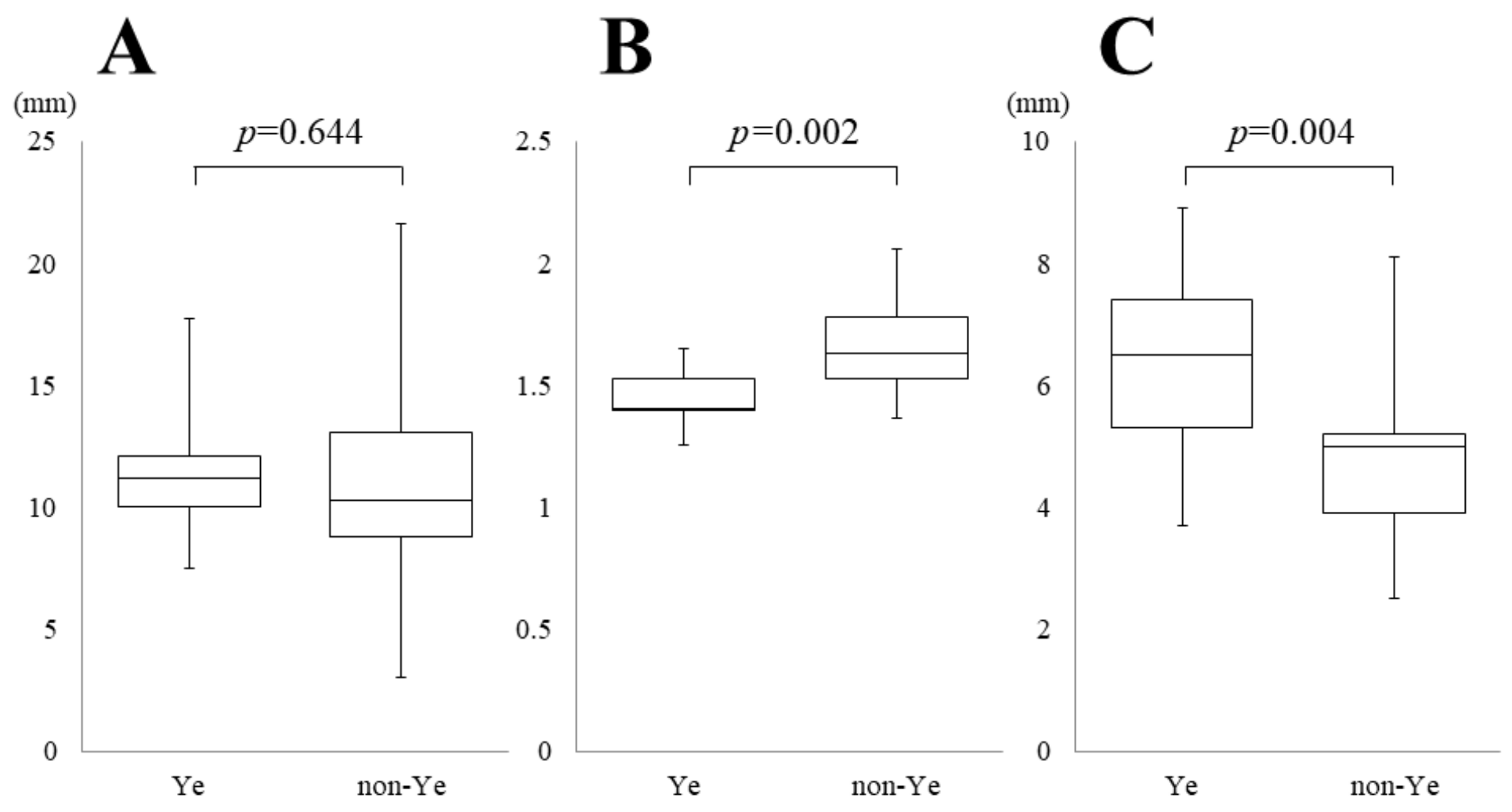

Figure 2

Comparison of measurements from abdominal ultrasonography between the two groups. a Maximum diameter of ileocecal lymph nodes; b Mean major/minor axis ratio of the ileocecal lymph node; $c$ Wall thickness of the terminal ileum 


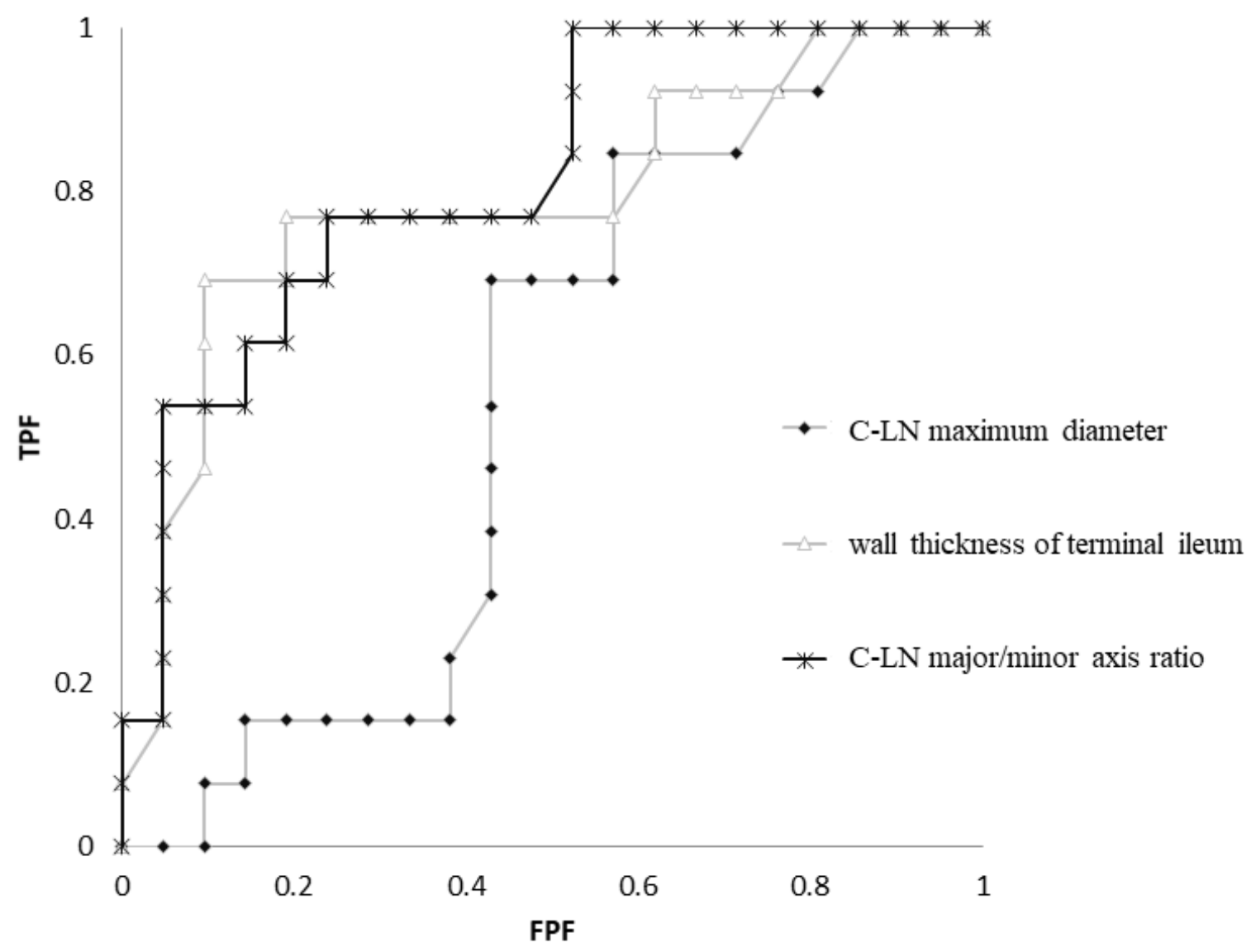

Figure 3

ROC curves for measurement values from abdominal ultrasonography. The ROC curves of the mean ileocecal lymph node major/minor axis ratio and the wall thickness of terminal ileum were significantly difference in two groups.

\section{Supplementary Files}

This is a list of supplementary files associated with this preprint. Click to download.

- BMCGtable1.xIsx

- BMCGadditionalfile1.xls.xlsx

- BMCGtable2.xIsx

- BMCGadditinalfile2.xls.xlsx 